\title{
Conical intersection and coherent vibrational dynamics in alkyl iodides captured by attosecond transient absorption spectroscopy
}

\author{
Kristina F. Chang, ${ }^{1}$ Han Wang, ${ }^{2}$ Sonia M. Poullain, ${ }^{3}$ Jesús González-Vázquez, ${ }^{4,5}$ Luis Bañares, ${ }^{3,6}$ David \\ Prendergast, $^{2,7}$ Daniel M. Neumark, ${ }^{1,2}$ and Stephen R. Leone ${ }^{1,2,8}$ \\ 1) Department of Chemistry, University of California, Berkeley, California 94720, USA. \\ ${ }^{2)}$ Chemical Sciences Division, Lawrence Berkeley National Laboratory, Berkeley, California 94720 , \\ USA. \\ ${ }^{3)}$ Departamento de Química Física, Facultad de Ciencias Químicas, Universidad Complutense de Madrid, Madrid 28040, \\ Spain. \\ 4) Departamento de Química, Universidad Autónoma de Madrid, Madrid 28049, Spain. \\ ${ }^{5)}$ Institute of Advanced Research on Chemistry, Universidad Autónoma de Madrid, 28049 Madrid, \\ Spain. \\ 6) Instituto Madrileño de Estudios Avanzados en Nanociencia (IMDEA-Nanoscience), Cantoblanco, 28049, Madrid, \\ Spain. \\ 7) Molecular Foundry, Lawrence Berkeley National Laboratory, Berkeley, California 94720, \\ USA. \\ ${ }^{8)}$ Department of Physics, University of California, Berkeley, California 94720, USA.
}

(*Electronic mail: srl@berkeley.edu.)

(*Electronic mail: dneumark@berkeley.edu.)

(Dated: 31 January 2022)

The photodissociation dynamics of alkyl iodides along the C-I bond are captured by attosecond extreme-ultraviolet (XUV) transient absorption spectroscopy employing resonant $\sim 20$ fs UV pump pulses. The methodology of previous experiments on $\mathrm{CH}_{3} \mathrm{I}$ [Chang, et al., J. Chem. Phys. 154, 234301 (2021)] is extended to the investigation of a C-I bond-breaking reaction in the dissociative $A$-band of $\mathrm{C}_{2} \mathrm{H}_{5} \mathrm{I}, i-\mathrm{C}_{3} \mathrm{H}_{7} \mathrm{I}$, and $t-\mathrm{C}_{4} \mathrm{H}_{9} \mathrm{I}$. Probing iodine $4 d$ core-to-valence transitions in the XUV enables one to map wave packet bifurcation at a conical intersection in the $A$-band as well as coherent vibrations in the ground state of the parent molecules. Analysis of spectroscopic bifurcation signatures yields conical intersection crossing times, found to be $15 \pm 4$ fs for $\mathrm{CH}_{3} \mathrm{I}, 14 \pm 5 \mathrm{fs}$ for $\mathrm{C}_{2} \mathrm{H}_{5} \mathrm{I}$, and $24 \pm 4$ fs for $i$ - $\mathrm{C}_{3} \mathrm{H}_{7} \mathrm{I}$ and $t-\mathrm{C}_{4} \mathrm{H}_{9} \mathrm{I}$. Observations of coherent vibrations, resulting from a projection of $A$-band structural dynamics onto the ground state by resonant impulsive stimulated Raman scattering (RISRS), indirectly reveal multimode C-I stretch and CCI bend vibrations in the $A$-band of $\mathrm{C}_{2} \mathrm{H}_{5} \mathrm{I}, i-\mathrm{C}_{3} \mathrm{H}_{7} \mathrm{I}$, and $t-\mathrm{C}_{4} \mathrm{H}_{9} \mathrm{I}$.

\section{INTRODUCTION}

Couplings between electronic and vibrational motions are a defining feature of photochemical reactions. ${ }^{1-4}$ The observation of electronic and vibrational evolution during a chemical reaction has therefore represented an important goal in spectroscopy. The detection of chemical dynamics with electronicstate and vibrational-mode specificity is especially important for understanding nonadiabatic conical intersection dynamics, in which the interplay of electronic and nuclear degrees of freedom is key in determining the course of a chemical reaction. ${ }^{1-7}$ Attosecond transient absorption spectroscopy (ATAS) is a powerful, recently developed experimental tool for investigating chemical dynamics. ${ }^{8}$ In ATAS, dynamics are tracked through the measurement of element-specific coreto-valence absorption transitions using isolated attosecond pulses in the extreme-ultraviolet (XUV). Due to the sensitivity of core-to-valence transitions to both the valence electronic structure and bonding structure of molecules, ultrafast XUV absorption spectroscopy has been successfully applied to the measurement of electronic state-switching dynamics at conical intersections and avoided crossings ${ }^{9-14}$ as well as of molecular vibrations with sub-picometer precision. ${ }^{15-17}$ In this study, the sensitivity of ATAS to electronic and nuclear dynamics enables the observation of conical intersection dy- namics in the $A$-band photodissociation of alkyl iodides (R-I, $\mathrm{R}=\mathrm{C}_{\mathrm{n}} \mathrm{H}_{\mathrm{m}}$ ).

The alkyl iodide $A$-band is accessed by UV excitation (230$290 \mathrm{~nm}$ ), involving a $5 p \rightarrow \sigma^{*}$ transition of a valence electron from a nonbonding iodine orbital into an antibonding orbital along the C-I bond. ${ }^{18,19}$ The excitation comprises one strong parallel transition to a ${ }^{3} \mathrm{Q}_{0}$ state and two weak perpendicular transitions to ${ }^{1} \mathrm{Q}_{1}$ and ${ }^{3} \mathrm{Q}_{1}$ states. ${ }^{20,21}$ For few-carbon containing alkyl iodides, excitation to ${ }^{3} \mathrm{Q}_{0}$ makes up $70-80 \%$ of the oscillator strength within the $A$-band..$^{22,23}$ Due to the repulsive character of the states along the C-I bond, UV excitation prompts rapid molecular fragmentation. ${ }^{24-26}$ As shown in Fig. 1a, dissociation along ${ }^{3} \mathrm{Q}_{0}$ correlates diabatically with the release of spin-orbit excited $\mathrm{I}^{*}\left({ }^{2} \mathrm{P}_{1 / 2}\right)$ atoms. However, a ${ }^{3} \mathrm{Q}_{0} /{ }^{1} \mathrm{Q}_{1}$ conical intersection located near the FranckCondon window for UV excitation allows for population transfer to ${ }^{1} \mathrm{Q}_{1}$, correlating with the release of ground-state $\mathrm{I}\left({ }^{2} \mathrm{P}_{3 / 2}\right)$ atoms. Consequently, the photoproduct branching ratio (I:I*) measured at the end of the dissociation reaction ${ }^{27-30}$ is believed to be mainly controlled by conical intersection dynamics.

The I:I* ratio following $A$-band dissociation varies drastically as a function of R-group structure. I:I* yields obtained for excitation wavelengths between $277-280 \mathrm{~nm}$ are summarized in Fig. 1b for alkyl iodides of varying R-group 


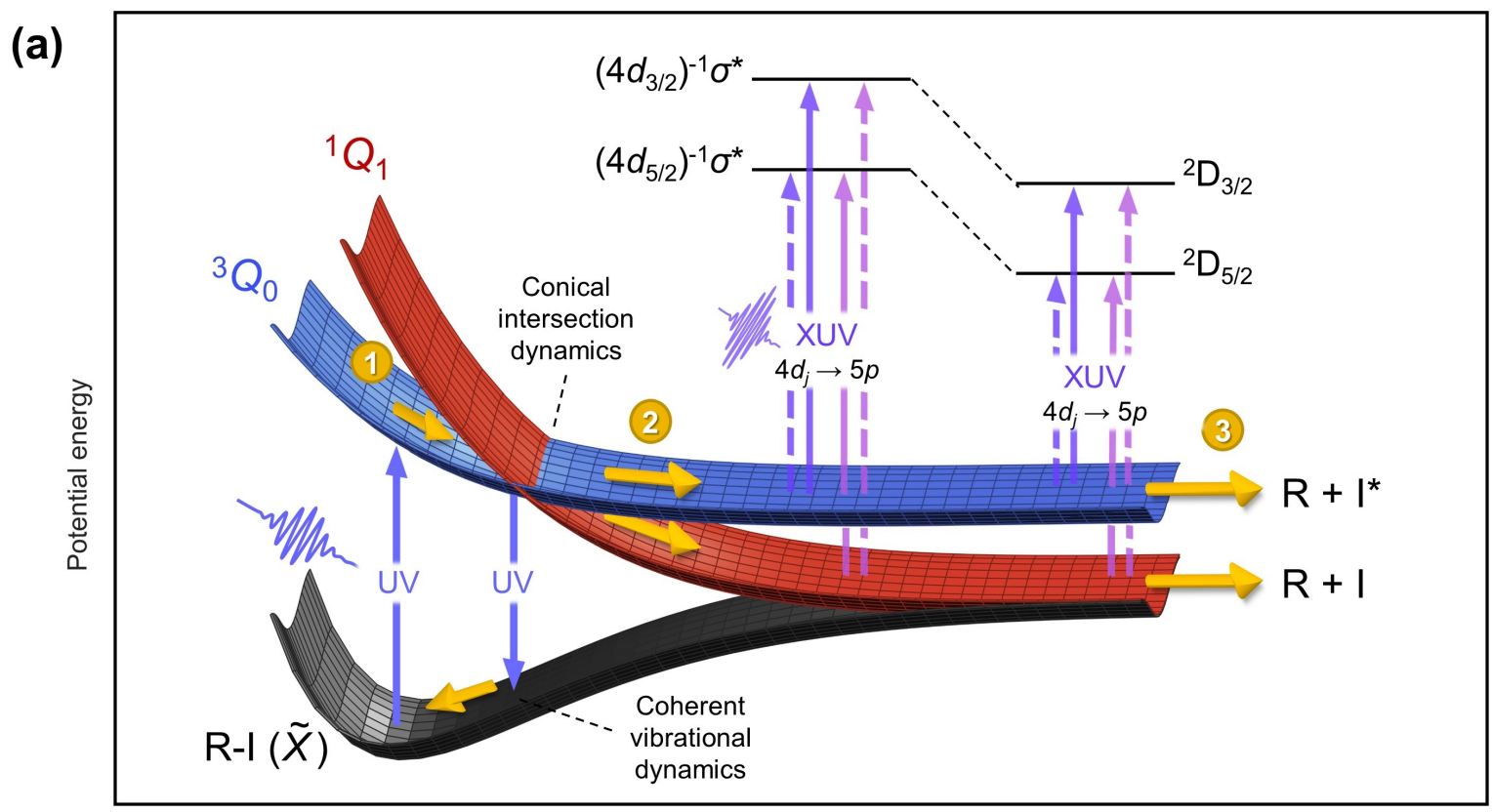

C-I bond length

(b)

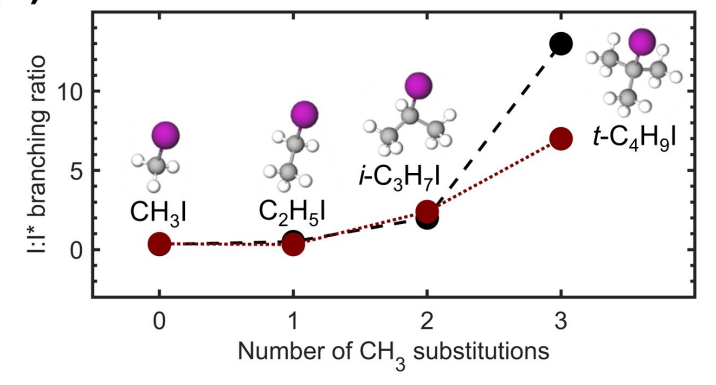

(c)

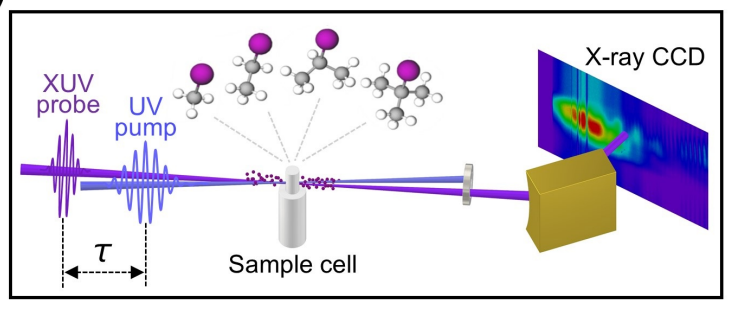

FIG. 1. Alkyl iodide dynamics and UV-XUV pump-probe concept. (a) Schematic of UV-induced dissociation through a conical intersection in the $A$-band and coherent vibrations in the ground state launched by resonant impulsive stimulated Raman scattering (RISRS). The potential energy surfaces are adapted from Ref. ${ }^{25}$, and are plotted as a function of C-I bond length and an orthogonal nuclear coordinate. In the $A$-band, $\mathrm{a}^{3} \mathrm{Q}_{0} /{ }^{1} \mathrm{Q}_{1}$ conical intersection allows for wave packet bifurcation into $\mathrm{I}^{*}$ and I dissociation channels. Dynamics along ${ }^{3} \mathrm{Q}_{0}$ and ${ }^{1} \mathrm{Q}_{1}$ before (Region 1), after the conical intersection (Region 2), and in the dissociation limit (Region 3) are probed by XUV transitions to core-excited $\left(4 d_{j}\right)^{-1} \sigma^{*}$ states, where $j=3 / 2$ or 5/2. Strong and weak XUV transitions are indicated with solid and dashed arrows, respectively. The XUV transition strengths of the ${ }^{3} \mathrm{Q}_{0}$ and ${ }^{1} \mathrm{Q}_{1}$ states are correlated with the strengths of atomic $\mathrm{I}^{*}$ and I transitions, respectively, which are governed by atomic selection rules. In the ground state, coherent vibrations are launched through the RISRS process consisting of UV excitation from the ground state, wave packet motion in the A-band, and UV-induced scattering back to the ground state (represented as a downwards UV arrow). For generality, the timing of the scattering step is drawn at an arbitrary time delay. The ground state is probed through XUV transitions (not pictured) to the $\left(4 d_{j}\right)^{-1} \sigma^{*}$ states. (b) Photoproduct I:I* ratios plotted as a function of $\mathrm{CH}_{3}$ substitution. I:I* ratios from narrowband 277-280 nm excitation ${ }^{27-30}$ (black circles) are compared with I:I* ratios from the current experiment (red circles). (c) Experimental setup.

structure. ${ }^{27-30}$ Additional methyl $\left(\mathrm{CH}_{3}\right)$ substitutions at the $\alpha$-carbon bring about a marked increase in the production of I atoms from the ${ }^{3} \mathrm{Q}_{0} /{ }^{1} \mathrm{Q}_{1}$ conical intersection. For methyl and ethyl iodide $\left(\mathrm{CH}_{3} \mathrm{I}\right.$ and $\left.\mathrm{C}_{2} \mathrm{H}_{5} \mathrm{I}\right)$, atomic I products are formed in a minority ratio of $1: 3$ relative to $\mathrm{I}^{*}{ }^{28,30}$ In contrast, the yield of I atoms in iso-propyl iodide $\left(i-\mathrm{C}_{3} \mathrm{H}_{7} \mathrm{I}\right)$ dominates with a I:I* ratio of $2: 1$, while tert-butyl iodide $\left(t-\mathrm{C}_{4} \mathrm{H}_{9} \mathrm{I}\right)$ provides an even greater ratio of 13:1. ${ }^{27,29}$ The significantlyenhanced production of I over I* atoms for branched, $\mathrm{CH}_{3}$ substituted molecules is interpreted as the result of a dramatic increase in ${ }^{3} \mathrm{Q}_{0} /{ }^{1} \mathrm{Q}_{1}$ state-switching probability at the conical intersection. ${ }^{24,27}$

Using ATAS with $\sim 50$ fs UV pump pulses, direct observations of conical intersection dynamics governing the I:I* branching ratios were previously obtained in $i-\mathrm{C}_{3} \mathrm{H}_{7} \mathrm{I}$ and $t$ $\mathrm{C}_{4} \mathrm{H}_{9} \mathrm{I}^{11}$ In subsequent experiments, improvements in the generation of $\sim 20 \mathrm{fs}$ UV pump pulses achieved the requisite time resolution to more definitively resolve conical intersection dynamics in $\mathrm{CH}_{3} \mathrm{I}^{12}$ Shortening the UV pulses allowed for the clear observation of wave packet bifurcation at the 
${ }^{3} \mathrm{Q}_{0} /{ }^{1} \mathrm{Q}_{1}$ conical intersection and enabled the identification of a conical intersection crossing time. In addition, coherent vibrational motion launched by resonant impulsive stimulated Raman scattering (RISRS) in the molecular $\mathrm{CH}_{3}$ I ground state was measured.

In this work, the dynamics previously observed in $\mathrm{CH}_{3} \mathrm{I}$ by ATAS with shorter UV pump pulses are compared to new measurements on $\mathrm{C}_{2} \mathrm{H}_{5} \mathrm{I}, i-\mathrm{C}_{3} \mathrm{H}_{7} \mathrm{I}$, and $t-\mathrm{C}_{4} \mathrm{H}_{9} \mathrm{I}$. Experimentally, the molecules are excited by $\sim 20$ fs UV pump pulses and probed by isolated attosecond XUV pulses that monitor changes in the $\mathrm{I}(4 d)$ core-level absorption spectra. The experimental results capture the wave packet bifurcation at the $A$-band conical intersection, enabling estimates of the conical intersection crossing time, as well as ground state RISRS dynamics that reveal vibrations along multiple modes of the molecule. Since the RISRS process is sensitive to dynamics on the excited state in the vicinity of the conical intersection, the observed ground state coherent vibrational dynamics suggest that nuclear motions near the conical intersection are multidimensional in character.

\section{METHODS}

\section{A. Experimental}

Liquid $\mathrm{CH}_{3} \mathrm{I}$ (99.5\% purity), $\mathrm{C}_{2} \mathrm{H}_{5} \mathrm{I}$ (99.5\% purity), $i-\mathrm{C}_{3} \mathrm{H}_{7} \mathrm{I}$ (99\% purity), and $t-\mathrm{C}_{4} \mathrm{H}_{9} \mathrm{I}$ (95\% purity) samples are obtained from Sigma-Aldrich. The sample target consists of a gas flow cell with a $3 \mathrm{~mm}$ long path length and $300 \mu \mathrm{m}$ diameter hole size. In each experiment, the cell is flowed with gaseous sample ( $\sim 5$ Torr) at room temperature. To initiate and probe dynamics in the sample, an laser setup utilizing femtosecond UV pump pulses and time-delayed attosecond $\mathrm{XUV}$ probe pulses is used.

The experimental setup is based on a Ti:Sapphire amplifier system that provides carrier-envelope phase-stable nearinfrared (NIR) laser pulses $(780 \mathrm{~nm}, 27 \mathrm{fs}, 1.9 \mathrm{~mJ}, 1 \mathrm{kHz})$. The NIR pulses are spectrally broadened in a stretched hollow-core fiber (1.5 m long, $400 \mu \mathrm{m}$ inner diameter, FewCycle Inc.) filled with 2.0 bar of neon gas. The pulses are then temporally compressed by chirped mirrors, a $2 \mathrm{~mm}$ thick ammonium dihydrogen phosphate crystal, and fused silica glass wedges, as described in Ref. ${ }^{31}$. Following compression, near transform-limited, few-cycle pulses $(\sim 550-950 \mathrm{~nm}, 3.8 \mathrm{fs}$, $0.8 \mathrm{~mJ}$ ) are obtained and used to drive high harmonic generation (HHG). For HHG, the few-cycle pulses are tightly focused into a gas flow cell in vacuum filled with argon, producing isolated attosecond XUV pulses (200 as, 40-70 eV, $1 \mathrm{~nJ}$ per pulse) via amplitude gating. The spectrum of the generated XUV pulses is included in the supplementary material. Following HHG, transmission of the XUV through a 300 $\mathrm{nm}$ thick aluminum filter removes residual NIR light from the beam path. The XUV beam is subsequently focused by a goldcoated toroidal mirror into the sample gas cell. As shown in Fig. 1c, the transmitted XUV spectrum is then detected by a home-built XUV spectrometer consisting of a concave grating and an X-ray CCD camera. Over the 44.0-60.0 eV range in which $\mathrm{I}(4 d)$ transitions are measured, the energy resolution of the spectrometer is estimated to be $25 \mathrm{meV}$.

Femtosecond UV pump pulses ( $277 \mathrm{~nm}, 6 \mathrm{~nm}$ bandwidth, $\sim 20$ fs transform-limited duration, 8.0-8.6 $\mu \mathrm{J}$ per pulse) are obtained through nonlinear frequency mixing. The generated pump spectrum, shown in the supplementary material, lies within the UV absorption band of the alkyl iodides, but is slightly red-shifted relative to the absorption band maxima located between $260-270 \mathrm{~nm} .^{18,19}$. Details of the UV pump setup can be found in the supplementary material. Briefly, UV pump pulses are generated by sum-frequency mixing between $400 \mathrm{~nm}$ pulses and broadband $700-950 \mathrm{~nm}$ pulses in a barium borate (BBO) crystal. To avoid dispersion of the UV pulses in air, all optics for the generation and delivery of the UV beam to the sample gas cell are contained within a high vacuum chamber. At the sample gas cell, the UV beam is focused to a $100 \mu \mathrm{m}$ diameter spot size and meets the XUV beam at a crossing angle of $0.7^{\circ}$.

Prior to each experiment, a measurement of the UV-XUV instrument response function and a calibration of pump-probe time delay is obtained using an in situ cross-correlation measurement in xenon. ${ }^{11,32}$ A summary of the cross-correlation results can be found in the supplementary material. The transient absorption experiments are characterized by a temporal resolution of 23-25 fs and a calibrated zero time delay with a precision of $\pm 4-5 \mathrm{fs}$.

Transient XUV absorption spectra for each molecular sample are recorded as changes in optical density $\triangle \mathrm{OD}$ $=-\log \left[I_{\mathrm{XUV}+\mathrm{UV}}(E, \tau) / I_{\mathrm{XUV}}(E)\right]$, where $I_{\mathrm{XUV}+\mathrm{UV}}(E, \tau)$ is the XUV spectrum of the sample recorded at the time delay $\tau$ following the UV pump and $I_{\mathrm{XUV}}(E)$ is the XUV spectrum of the sample recorded in the absence of the pump. The integration times and frame averages used to produce each molecular transient are summarized in the supplementary material. The transients are collected at 4-fs intervals over a photon energy range between 40-60 eV. The $\mathrm{CH}_{3} \mathrm{I}$ and $\mathrm{C}_{2} \mathrm{H}_{5} \mathrm{I}$ transients are collected over a $\sim-60-300$ fs time delay range, and the $i-\mathrm{C}_{3} \mathrm{H}_{7} \mathrm{I}$ and $t-\mathrm{C}_{4} \mathrm{H}_{9} \mathrm{I}$ transients are collected over a $\sim-60$ 500 fs time delay range. Finally, the raw transients are postprocessed to eliminate high-frequency noise using a low-pass Gaussian filter. Raw transients plotted over the full photon energy range recorded can be found in the supplementary material.

\section{B. Theory}

Calculations used to produce the simulated $\mathrm{CH}_{3} \mathrm{I}$ transients are used for comparison to the experimental ATAS results, and have been previously published in Ref. ${ }^{33}$. To produce the simulated transients, nonadiabatic dynamics of $\mathrm{CH}_{3} \mathrm{I}$ following excitation to the ${ }^{3} \mathrm{Q}_{0}$ state were computed with Tully's fewestswitches surface hopping theory (FSSH) in the SHARC software package. XUV transient absorption spectra of the molecular dynamics trajectories obtained from these calculations were then computed using the MS-CASPT2 method in OpenMolcas with the ANO-RCC-VTZP basis set. Distinct XUV transients corresponding to dissociative trajectories producing 
I and $\mathrm{I}^{*}$ are obtained, and modified to the produce simulated transients capturing measured I:I* branching ratios. To enhance comparisons to the experimental results, a 20 fs temporal broadening is applied to the computed transients in order to better simulate the temporal resolution of the experiment.

\section{RESULTS AND DISCUSSION}

State-specific dissociation dynamics of the alkyl iodides in the $A$-band and vibrational dynamics in the ground state are probed in the XUV through $\mathrm{I}(4 d)$ core-to-valence excitations. As shown in Fig. 1(a), the probing scheme maps wave packet motion from the repulsive regions of the ${ }^{3} \mathrm{Q}_{0}$ and ${ }^{1} \mathrm{Q}_{1}$ potentials before and after the conical intersection $(\mathrm{Re}-$ gions 1-2) out to the dissociation limit (Region 3). The ${ }^{3} \mathrm{Q}_{0}$ and ${ }^{1} \mathrm{Q}_{1}$ states are characterized by the configuration $(4 d)^{10} \ldots$ $(\sigma)^{2}(5 p)^{3}\left(\sigma^{*}\right)^{1}$, correlating to I and I* atomic limits characterized by the configuration $(4 d)^{10} \ldots(5 p)^{5} .{ }^{3} \mathrm{Q}_{0}$ and ${ }^{1} \mathrm{Q}_{1}$ fragmentation can therefore be followed through the probing of available $4 d_{j} \rightarrow 5 p$ molecular transitions converging to an asymptotic dissociation limit characterized by well-known $4 d_{j} \rightarrow 5 p$ transitions of $\mathrm{I}^{*}$ and I where $j=3 / 2$ or $5 / 2 .{ }^{32}$ Due to the large $\sim 1.7 \mathrm{eV}$ spin-orbit splitting of the $4 d_{j}$ core hole, atomic and molecular features associated with $4 d_{5 / 2} \rightarrow 5 p$ and $4 d_{3 / 2} \rightarrow 5 p$ transitions are separated in energy into respective 44.5-46.3 eV and 46.3-48.5 eV spectral regions. ${ }^{11,33}$ In each XUV spectral region, wave packet bifurcation at the conical intersection and dissociation dynamics are mapped onto bifurcation signatures of a ${ }^{3} \mathrm{Q}_{0}$ (Region 1) feature into the ${ }^{3} \mathrm{Q}_{0}$ and ${ }^{1} \mathrm{Q}_{1}$ (Region 2) features converging to well-defined $\mathrm{I}^{*}$ and I peaks in the dissociation limit. Finally, the molecular ground state is probed through pairs of $4 d_{j} \rightarrow \sigma^{*}$ transitions, and coherent wave packet motion in the ground state is mapped to periodic photon energy oscillations in the corresponding spectral features. ${ }^{16}$

Two-dimensional colormaps depicting transient absorption spectra from 44.5-53.5 eV for each molecule are plotted in Fig. 2. The 44.5-49.5 eV spectral region of each transient is dominated by positive $\Delta \mathrm{OD}$ features characterizing molecular excited states and atomic photoproducts probed through $4 d_{j} \rightarrow 5 p$ transitions. Meanwhile, the $49.5-53.5 \mathrm{eV}$ spectral region is dominated by a pair of oscillating negative $\Delta \mathrm{OD}$ features that capture coherent vibrations in the molecular ground state through $4 d_{j} \rightarrow \sigma^{*}$ transitions. ${ }^{16}$ In the following sections, we (i) elucidate spectral signatures of $A$-band conical intersection and dissociation dynamics and (ii) interpret the vibrational coherences in the ground state.

\section{A. A-band photodissociation and conical intersection dynamics}

Expanded views of the transients in the 44.5-48.5 spectral region where $A$-band dynamics are captured are shown in Figs. 3(a)-(b) for $\mathrm{CH}_{3} \mathrm{I}$ and $\mathrm{C}_{2} \mathrm{H}_{5} \mathrm{I}$ and 4(a)-(b) for $i-\mathrm{C}_{3} \mathrm{H}_{7} \mathrm{I}$ and $t-\mathrm{C}_{4} \mathrm{H}_{9} \mathrm{I}$. The transients can be subdivided into 44.5-46.3 $\mathrm{eV}$ and 46.3-48.5 eV spectral regions associated with $4 d_{5 / 2} \rightarrow$
$5 p$ and $4 d_{3 / 2} \rightarrow 5 p$ transitions, respectively. In each molecular transient, the convergence of short-lived features at early time delays (0-100 fs) into the fixed values of purely-atomic iodine transitions at long time delays (100-200 fs) provides a map of C-I bond-breaking from repulsive regions of the $A$ band potentials (Regions 1-2, Fig. 1(a)) into the asymptotic dissociation limit (Region 3, Fig. 1(a)).

We first discuss the purely-atomic iodine transitions observed in the transients at long time delays. Atomic I and $\mathrm{I}^{*}$ produced in the dissociation limit (Region 3, Fig. 1(a)) are characterized by three allowed $45.9 \mathrm{eV}\left[\mathrm{I}\left({ }^{2} \mathrm{P}_{3 / 2} \rightarrow\right.\right.$ $\left.\left.{ }^{2} \mathrm{D}_{5 / 2}\right)\right], 46.7 \mathrm{eV}\left[\mathrm{I} *\left({ }^{2} \mathrm{P}_{1 / 2} \rightarrow{ }^{2} \mathrm{D}_{3 / 2}\right)\right]$, and $47.6 \mathrm{eV}\left[\mathrm{I}\left({ }^{2} \mathrm{P}_{3 / 2}\right.\right.$ $\left.\left.\rightarrow{ }^{2} \mathrm{D}_{3 / 2}\right)\right]$ transitions and a forbidden $45.0 \mathrm{eV}\left[\mathrm{I}^{*}\left({ }^{2} \mathrm{P}_{1 / 2} \rightarrow\right.\right.$ $\left.{ }^{2} \mathrm{D}_{5 / 2}\right)$ ] transition. ${ }^{34,35}$ In the $\mathrm{CH}_{3} \mathrm{I}$ transient, the rise of the $\mathrm{I}^{*}$ signals within $100 \mathrm{fs}$ is consistent with the sub-100 fs C-I dissociation in the $A$-band. ${ }^{32}$ The atomic I signal continues to evolve beyond $100 \mathrm{fs}$, in contrast to the simulated spectrum (Fig. 3(c)), indicating contributions from multiphoton UV excitation. ${ }^{12}$ The dissociation limit is reached within 100 fs for $\mathrm{C}_{2} \mathrm{H}_{5} \mathrm{I}$, and within 200 fs for $i-\mathrm{C}_{3} \mathrm{H}_{7} \mathrm{I}$ and $t-\mathrm{C}_{4} \mathrm{H}_{9} \mathrm{I}$, and is consistent with previously-measured fragmentation times obtained from single-photon dissociation. ${ }^{25}$ Finally, the photoproduct $\mathrm{I}: \mathrm{I}^{*}$ ratio of each molecule is determined based on the relative intensities of atomic I and I* transitions. The I:I* ratios associated with single-photon, $A$-band dissociation is observed here to be $\sim 1: 3$ for $\mathrm{CH}_{3} \mathrm{I}$ and $\mathrm{C}_{2} \mathrm{H}_{5} \mathrm{I}, \sim 2: 1$ for $i$ $\mathrm{C}_{3} \mathrm{H}_{7} \mathrm{I}$ and $\sim 7: 1 t-\mathrm{C}_{4} \mathrm{H}_{9} \mathrm{I}$ (see supplementary material). For $\mathrm{CH}_{3} \mathrm{I}$, the I:I* ratio takes into account contributions from multiphoton excitation. As shown in Fig. 1b, the experimentally estimated I:I* ratios are roughly consistent with previous measurements resulting from narrowband UV excitation. ${ }^{27-30}$ The I:I* ratios have been demonstrated to be dependent on the wavelength of the UV pulse used, ${ }^{36}$ and differences in the measured I:I* ratios of this study compared to previous experiments may arise from the use of a broadband UV pump in the present work.

At early time delays in the transients (Figs. 3(a)-(b), 4(a)(b)), transient molecular features reveal conical intersection dynamics preceding C-I dissociation. Signatures of $A$-band conical intersections are verified through comparisons to modified simulations (Figs. 3(c), 4(c)-(d)) from Ref. ${ }^{33}$ based on a $\mathrm{CH}_{3} \mathrm{I}$ model system. To facilitate comparisons to each $\mathrm{CH}_{3} \mathrm{I}, \mathrm{C}_{2} \mathrm{H}_{5} \mathrm{I}, i-\mathrm{C}_{3} \mathrm{H}_{7} \mathrm{I}$, and $t-\mathrm{C}_{4} \mathrm{H}_{9} \mathrm{I}$ measurement, the simulated $\mathrm{CH}_{3} \mathrm{I}$ transients in Figs. 3 and 4 are modified to reflect the experimentally-observed $\mathrm{I}: \mathrm{I}^{*}$ ratios in the dissociation limit. In the case of $\mathrm{CH}_{3} \mathrm{I}$, the experimental ratio associated with single-photon dynamics is used. In Fig. 3, the simulation converges to the $\mathrm{CH}_{3} \mathrm{I}$ and $\mathrm{C}_{2} \mathrm{H}_{5} \mathrm{I}$ photoproduct $\mathrm{I}: \mathrm{I}^{*}$ ratio of $\sim 1: 3$, reflecting dissociation dynamics that follow predominantly from diabatic dissociation on ${ }^{3} \mathrm{Q}_{0}$. Meanwhile, Fig. 4 shows modified simulations converging to $i-\mathrm{C}_{3} \mathrm{H}_{7} \mathrm{I}$ and $t-\mathrm{C}_{4} \mathrm{H}_{9} \mathrm{I}$ photoproduct $\mathrm{I}: \mathrm{I}^{*}$ ratios of $\sim 2: 1$ and $\sim 7: 1$, reflecting dissociation dynamics that follow predominantly from nonadiabatic state-switching to the ${ }^{1} \mathrm{Q}_{1}$ state at the conical intersection. In the subsequent discussion, qualitative comparisons between the experimental and simulated transients allow for the assignment of electronic state-specific dissociation and conical intersection dynamics in the $A$-band. 

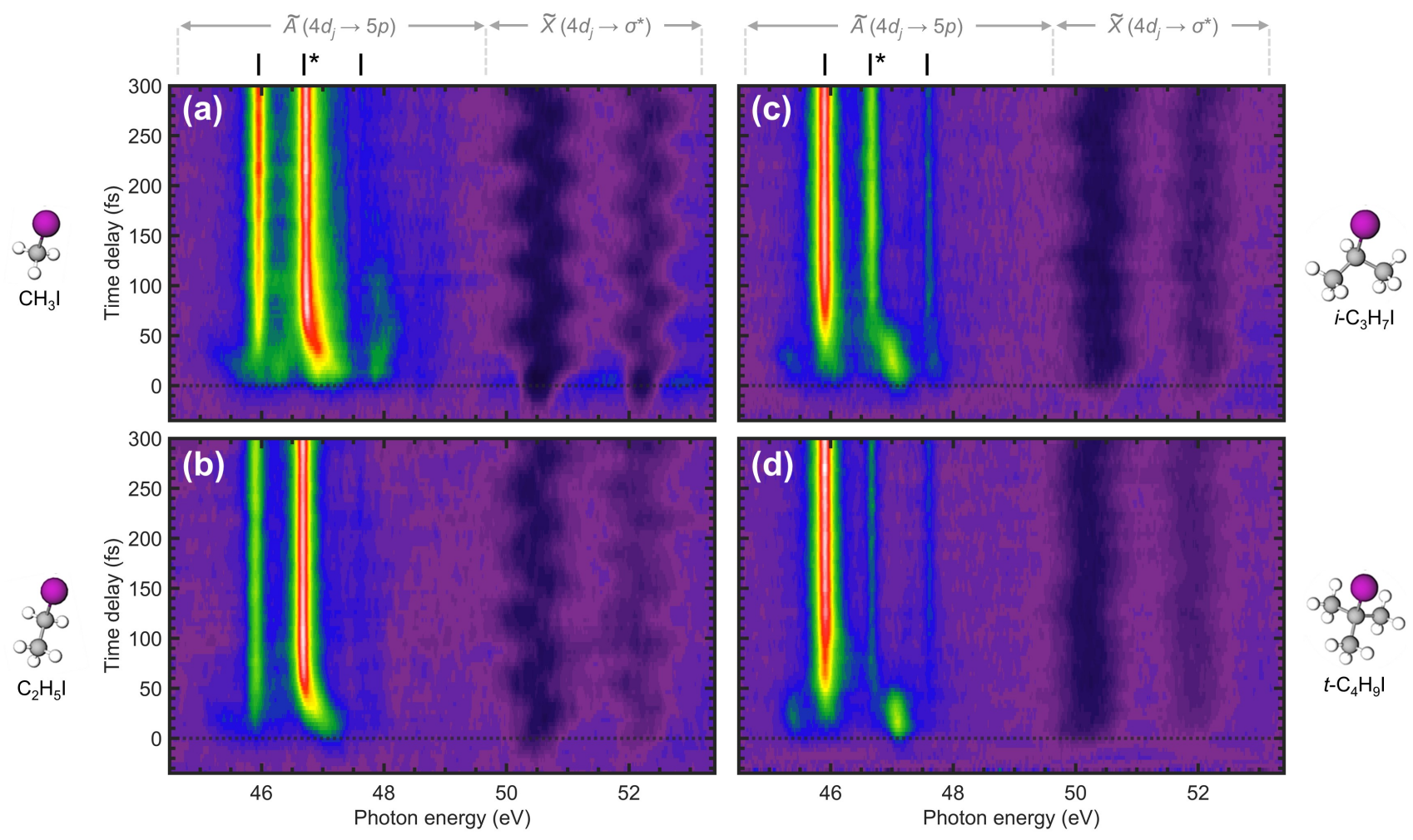

$-0.1$

$-0.05$

0

0.05

$\frac{\mathbf{L}}{0.1}$

0.15

0.2

FIG. 2. Experimental transient absorption spectra of (a) $\mathrm{CH}_{3} \mathrm{I}$, (b) $\mathrm{C}_{2} \mathrm{H}_{5} \mathrm{I}$, (c) $i$ - $\mathrm{C}_{3} \mathrm{H}_{7} \mathrm{I}$, and (d) $t$ - $\mathrm{C}_{4} \mathrm{H}_{9} \mathrm{I}$. In the $44.5-48.5$ eV spectral region, $A$-band photodissociation dynamics are observed through $4 d \rightarrow 5 p$ transitions. The locations of well-known atomic iodine transitions are labelled above each transient. In the 48.5-53.5 eV spectral region, coherent vibrational dynamics in the molecular ground state are mapped through $4 d \rightarrow \sigma^{*}$ transitions. The colorscales of the transients are plotted in $\Delta \mathrm{OD}$, shown at the bottom. The $\mathrm{CH}_{3} \mathrm{I}$ results are previously published in Ref. ${ }^{12}$, but are included here for comparison to new results in $\mathrm{C}_{2} \mathrm{H}_{5} \mathrm{I}, i-\mathrm{C}_{3} \mathrm{H}_{7} \mathrm{I}$, and $t-\mathrm{C}_{4} \mathrm{H}_{9} \mathrm{I}$.

$A$-band dissociation and conical intersection dynamics in the experimental and simulated transients (Figs. 3 and 4) show strong qualitative similarities. As illustrated in the XUV probing scheme of Fig. 1(a), $A$-band dissociation dynamics are mapped through the rise of initial ${ }^{3} \mathrm{Q}_{0}$ (Region 1) features capturing direct UV pump excitation to the ${ }^{3} \mathrm{Q}_{0}$ state above the conical intersection at $0 \mathrm{fs}$ time delay, the bifurcation of the ${ }^{3} \mathrm{Q}_{0}$ features into distinct ${ }^{3} \mathrm{Q}_{0}$ and ${ }^{1} \mathrm{Q}_{1}$ (Region 2) features after the conical intersection, observed between $\sim 10-30$ $\mathrm{fs}$, and the asymptotic convergence of state-specific molecular features after the conical intersection (Region 2) into atomic I and I* dissociation limits (Region 3) after $\sim 80-100$ fs. Statespecific molecular features capturing these dynamics through $4 d_{5 / 2} \rightarrow 5 p$ and $4 d_{3 / 2} \rightarrow 5 p$ transitions in the $44.5-46.3 \mathrm{eV}$ and $46.3-48.5 \mathrm{eV}$ spectral regions, respectively, are labelled on the experimental and simulated transients in Figs. 3 and 4. Observed photon energy shifts of initial ${ }^{3} \mathrm{Q}_{0}$ (Region 1) molecular features and their bifurcation into ${ }^{3} \mathrm{Q}_{0}$ and ${ }^{1} \mathrm{Q}_{1}$ (Region 2) features after the conical intersection are denoted by arrows on the experimental and theoretical transients. While the majority of the simulated shifts are experimentally observed, signal-to-noise limitations in the experiments preclude the observation of a few of the theoretically-predicted shifts indi- cated by dashed arrows. In particular, a weak ${ }^{1} \mathrm{Q}_{1}$ (Region 2) feature correlating to the weak $\mathrm{I}(47.6 \mathrm{eV})$ atomic transition is challenging to resolve in the $\mathrm{CH}_{3} \mathrm{I}$ and $\mathrm{C}_{2} \mathrm{H}_{5} \mathrm{I}$ transients (Fig. 3). However, this ${ }^{1} \mathrm{Q}_{1}$ (Region 2) feature is observable in the experimental $i-\mathrm{C}_{3} \mathrm{H}_{7} \mathrm{I}$ and $t-\mathrm{C}_{4} \mathrm{H}_{9} \mathrm{I}$ transients (Fig. 4) owing to the higher probability of electronic state-switching in these systems. For the same reason, a weak ${ }^{3} \mathrm{Q}_{0}$ (Region 2) feature correlating to the forbidden $\mathrm{I}^{*}(45.0 \mathrm{eV})$ limit observed in $\mathrm{CH}_{3} \mathrm{I}$ and $\mathrm{C}_{2} \mathrm{H}_{5} \mathrm{I}$ is not observable in $i-\mathrm{C}_{3} \mathrm{H}_{7} \mathrm{I}$ and $t-\mathrm{C}_{4} \mathrm{H}_{9} \mathrm{I}$. Instead, conical intersection dynamics are observed through bifurcation signatures appearing in the $4 d_{5 / 2} \rightarrow 5 p$ transitions in the 44.5-46.3 eV spectral region for $\mathrm{CH}_{3} \mathrm{I}$ and $\mathrm{C}_{2} \mathrm{H}_{5} \mathrm{I}$, and through and $4 d_{3 / 2} \rightarrow 5 p$ transitions in $46.3-48.5 \mathrm{eV}$ spectral region for $i-\mathrm{C}_{3} \mathrm{H}_{7} \mathrm{I}$ and $t-\mathrm{C}_{4} \mathrm{H}_{9} \mathrm{I}$.

Observations of conical intersection bifurcations in the 44.5-46.3 eV spectral region for $\mathrm{CH}_{3} \mathrm{I}$ and $\mathrm{C}_{2} \mathrm{H}_{5} \mathrm{I}$ and in the 46.3-48.5 eV spectral region for $i-\mathrm{C}_{3} \mathrm{H}_{7} \mathrm{I}$ and $t-\mathrm{C}_{4} \mathrm{H}_{9} \mathrm{I}$ are shown in Fig. 5. From the bifurcation signatures, conical intersection crossing times are estimated. As discussed in previous work on $\mathrm{CH}_{3} \mathrm{I},{ }^{12}$, the bifurcation of a ${ }^{3} \mathrm{Q}_{0}$ (Region 1) wave packet into ${ }^{3} \mathrm{Q}_{0}$ and ${ }^{1} \mathrm{Q}_{1}$ (Region 2) wave packets at the conical intersection forms a u-shaped spectral feature converging toward atomic I* and I limits at long time delays. The min- 

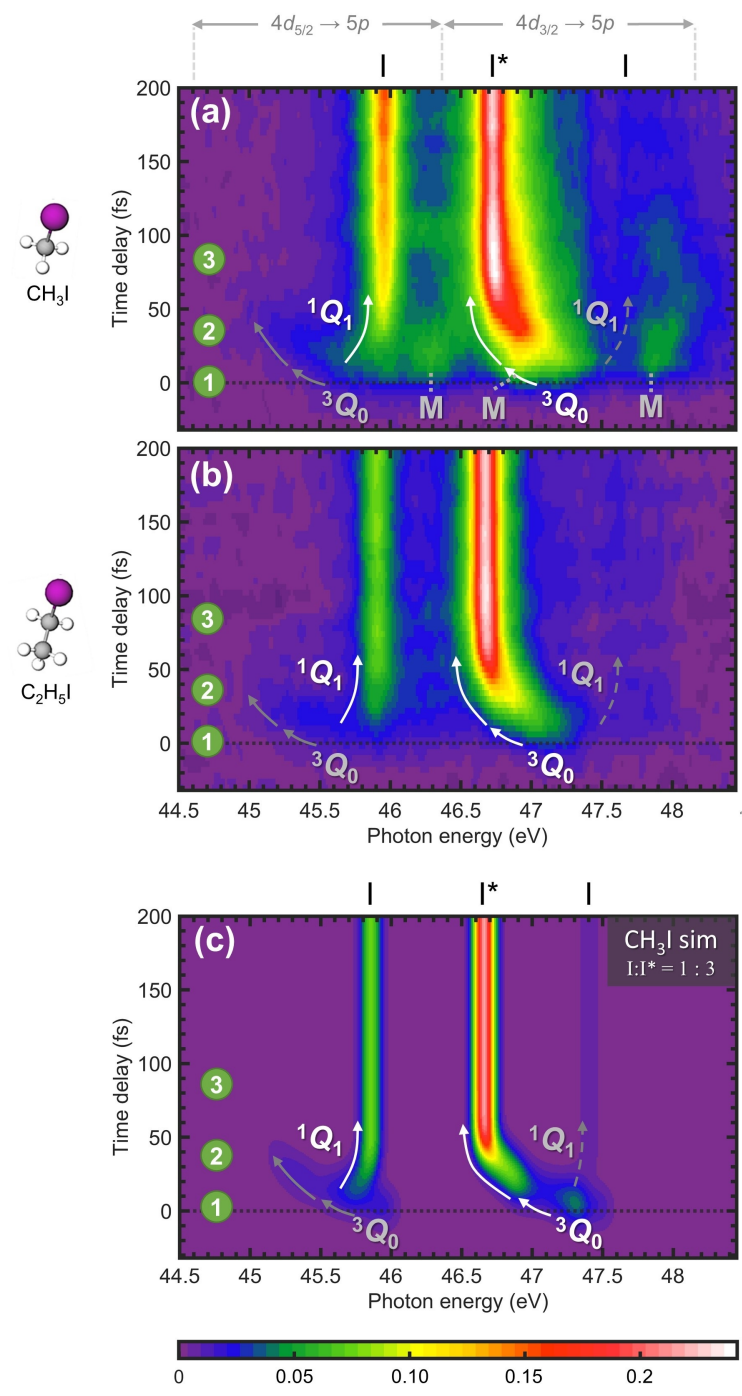

FIG. 3. Experimental $\mathrm{CH}_{3} \mathrm{I}$ and $\mathrm{C}_{2} \mathrm{H}_{5} \mathrm{I}$ transients and modified $\mathrm{CH}_{3} \mathrm{I}$ simulations representing an I:I* ratio of 1:3. The experimental (a) $\mathrm{CH}_{3} \mathrm{I}$ and (b) $\mathrm{C}_{2} \mathrm{H}_{5} \mathrm{I}$ transients are compared to $\mathrm{CH}_{3} \mathrm{I}$ simulations presented in panel (c). The simulation is temporally broadened by a Gaussian and independently normalized for comparison to the experiments. The $4 d_{5 / 2} \rightarrow 5 p$ spectral region $(44.5-46.3 \mathrm{eV})$ and $4 d_{3 / 2} \rightarrow$ $5 p$ spectral region (46.3-48.5 eV) are labelled above each transient. Experimentally, wave packet bifurcation at the conical intersection is most clearly resolved in the $4 d_{5 / 2} \rightarrow 5 p$ spectral region of the transients. In panel (a), the assignment of multiphoton features, labeled $\mathrm{M}$ in the experimental $\mathrm{CH}_{3} \mathrm{I}$ results, is described in Ref. ${ }^{12}$.

imum of the u-shaped features corresponds to the temporal origin of the wave packet bifurcation event and is interpreted as the conical intersection crossing time. In Fig. 5, the centroid of the u-shaped features observable in the $4 d_{5 / 2} \rightarrow 5 p$ spectral region (44.5-46.3 eV) for $\mathrm{CH}_{3} \mathrm{I}$ and $\mathrm{C}_{2} \mathrm{H}_{5} \mathrm{I}$ and in the $4 d_{3 / 2} \rightarrow 5 p$ spectral region $(46.3-48.5 \mathrm{eV})$ for $i-\mathrm{C}_{3} \mathrm{H}_{7} \mathrm{I}$ and $t-\mathrm{C}_{4} \mathrm{H}_{9} \mathrm{I}$ are extracted as a function of photon energy and superimposed on the transients. Conical intersection crossing times are then extracted as the minima of a spline trace (red curve) taken along these points. The resulting crossing times are summarized in Table I. Based on the results, the four alkyl iodides are found to undergo conical intersection dynamics within 30 fs. The crossing times of the simpler $\mathrm{CH}_{3} \mathrm{I}$ and $\mathrm{C}_{2} \mathrm{H}_{5}$ I molecules are estimated to be $\sim 14-15 \mathrm{fs}$, while the estimated crossings times of the more highly substituted $i-\mathrm{C}_{3} \mathrm{H}_{7} \mathrm{I}$ and $t-\mathrm{C}_{4} \mathrm{H}_{9} \mathrm{I}$ molecules are found to be $\sim 24 \mathrm{fs}$. A small increase in the crossing times from $\mathrm{CH}_{3} \mathrm{I}$ and $\mathrm{C}_{2} \mathrm{H}_{5} \mathrm{I}$ to $i-\mathrm{C}_{3} \mathrm{H}_{7} \mathrm{I}$ and $t-\mathrm{C}_{4} \mathrm{H}_{9} \mathrm{I}$ suggests that the heavier and more highly substituted molecules require slightly more time to propagate from the Franck-Condon region to the conical intersection.

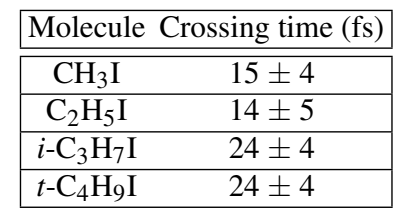

TABLE I. Experimentally-measured conical intersection crossing times. Crossing times are estimated from an analysis of the wave packet bifurcation signatures shown in Fig. 5. The uncertainty in the extracted crossing times is taken as the experimental uncertainty in the zero time delay of the experiment (see supplementary material).

\section{B. Ground state vibrational dynamics launched by resonant impulsive stimulated Raman scattering}

As observed in previous XUV transient absorption experiments, ${ }^{9}, 10,12,15,16$ ground state vibrational wave packet dynamics manifest as cosinusoidal photon energy shifts of ground state transient absorption signals. In the experimental transients (Fig. 2), cosinusoidal oscillations observed in the $4 d_{j} \rightarrow \sigma^{*}$ depletion signals provide a map of coherent vibrations launched in the molecular ground state by the UV pump. Vibrational dynamics are produced by a resonant UV pump interaction involving the $A$-band. Since the UV pump is both shorter in duration than the period of a typical alkyl iodide vibrational mode and resonant with transitions between the ground state and $A$-band, the origin of the observed ground state dynamics is ascribed to a two-photon process known as resonant impulsive stimulated Raman scattering (RISRS). ${ }^{37-41}$

A schematic of the RISRS mechanism is shown in Fig. 1(a). RISRS is modeled as a three-step process occurring within the duration of the UV pulse. In the first step, a UV photon excites a wave packet from the ground state into the ${ }^{3} \mathrm{Q}_{0}$ state. In the second step, the wave packet propagates along the ${ }^{3} \mathrm{Q}_{0}$ surface for the duration of the UV pulse, and partially bifurcates onto the ${ }^{1} \mathrm{Q}_{1}$ surface at the ${ }^{3} \mathrm{Q}_{0} /{ }^{1} \mathrm{Q}_{1}$ conical intersection. In the third and final step, a portion of the displaced wave packets from either excited state are projected to the ground state via scattering by a second, resonant UV photon. With a sufficiently short UV pulse, the scattering process generates a coherent superposition of vibrational levels constituting a periodically-oscillating wave packet in the ground state. Crucially, the vibrational modes along which the ground state wave packet oscillates depend on the nuclear coordi- 


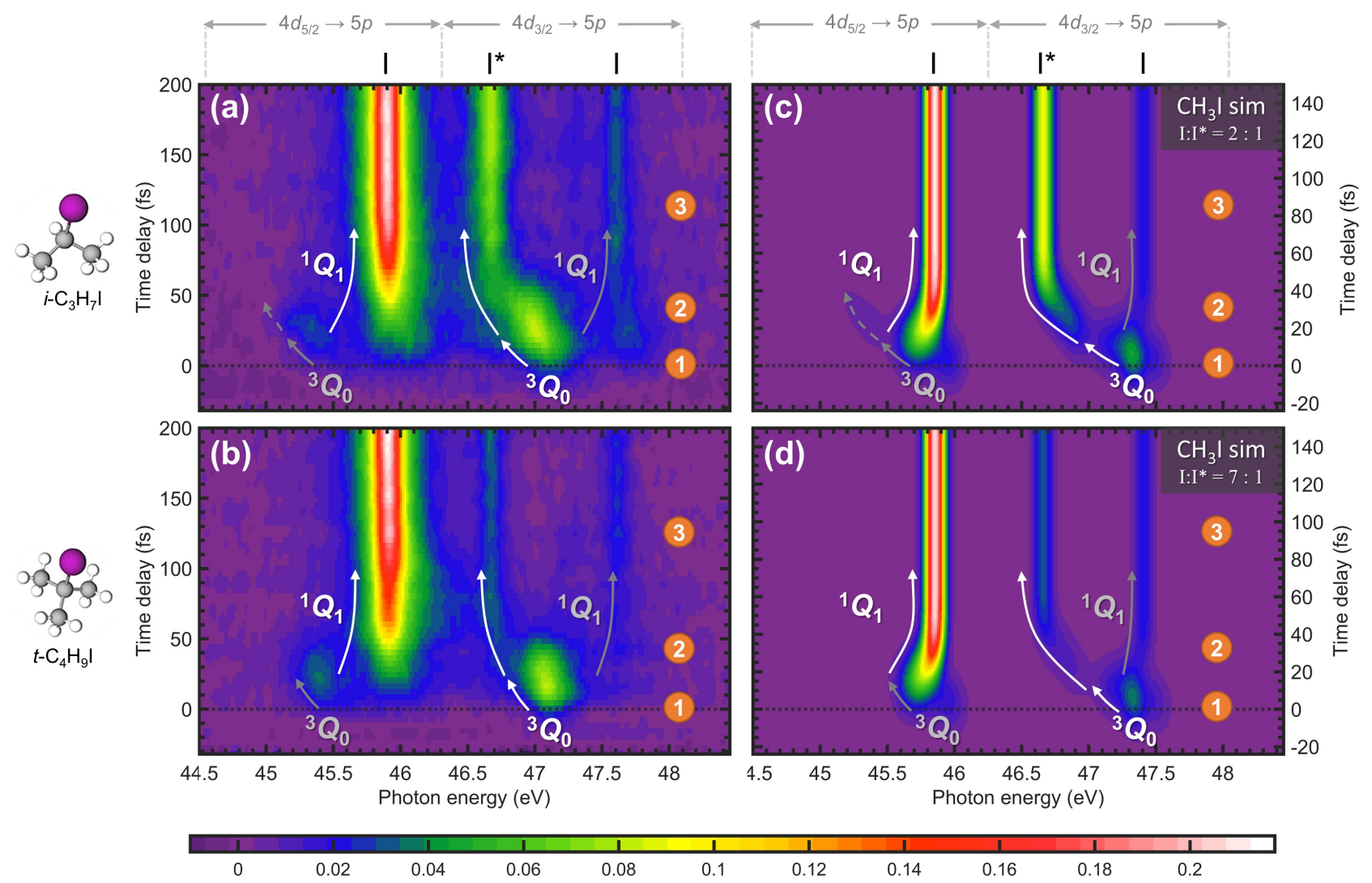

FIG. 4. Experimental $i$ - $\mathrm{C}_{3} \mathrm{H}_{7} \mathrm{I}$ and $t$ - $\mathrm{C}_{4} \mathrm{H}_{9} \mathrm{I}$ transients and modified $\mathrm{CH}_{3} \mathrm{I}$ simulations. Experimental (a) $i$ - $\mathrm{C}_{3} \mathrm{H}_{7} \mathrm{I}$ and (b) $t$ - $\mathrm{C}_{4} \mathrm{H}_{9} \mathrm{I}$ transients are compared to modified $\mathrm{CH}_{3} \mathrm{I}$ simulations presented in panels (c)-(d) converging to experimentally-observed I:I* branching ratios. The simulations are temporally broadened by a Gaussian and independently normalized for comparison to the experiments. The $4 d_{5 / 2} \rightarrow 5 p$ spectral region (44.5-46.3 eV) and $4 d_{3 / 2} \rightarrow 5 p$ spectral region (46.3-48.5 eV) are labelled above each transient. Experimentally, wave packet bifurcation at the conical intersection is most clearly resolved in the $4 d_{3 / 2} \rightarrow 5 p$ spectral region. Because the simulated $\mathrm{CH}_{3} \mathrm{I}$ transient evolves more quickly compared to the experimental $i-\mathrm{C}_{3} \mathrm{H}_{7} \mathrm{I}$ and $t-\mathrm{C}_{4} \mathrm{H}_{9} \mathrm{I}$ transients, the simulated transient is plotted over a smaller temporal range for easier qualitative comparison to the experimental results.

nates of excited state motion during the second step of RISRS. Ground state RISRS dynamics, therefore, have the potential to indirectly reveal ultrafast structural changes in the $A$-band on timescales dictated by the time delay of the scattering event. The ground state dynamics in the present experiments reflect RISRS scattering pathways from a distribution of time delays. Although the exact delays are unknown, the RISRS mechanism necessitates that the distribution is gated to the duration of the UV pulses ( $\sim 20 \mathrm{fs})$. The excited state motion implied by RISRS ground state dynamics are, therefore, representative of dynamics occurring before or just after the conical intersection encountered on the $\sim 14-24$ fs timescale (Fig. 1a, Regions 1-2), with minimal contributions beyond this timeframe. The measurement of the ground state wave packets therefore provides, in principle, an indirect means for detecting molecular structure dynamics associated with excited state dynamics approaching or just after the conical intersection. Finally, we note that if the scattering time delay occurs after the conical intersection, the ground state wave packets may reflect dynamics from either or both ${ }^{3} \mathrm{Q}_{0}$ and ${ }^{1} \mathrm{Q}_{1}$ surfaces.

Insight into the launching mechanism and vibrational mode composition of the RISRS wave packets is enabled by an analysis of the cosinusoidal oscillations within the ground state signals. Fig. 6 shows an expanded view of the strongest ground state signal of each molecule, corresponding to the $4 d_{5 / 2} \rightarrow \sigma^{*}$ transition. The oscillating positions of the ground state signals are determined by fitting the signals to a Gaussian spectral profile at each time delay, and tracking the evolution of the Gaussian center energy over time. The motion of the spectral profile in time, indicated with white circles in Fig. 6, is then modeled by a sum of cosines:

$$
E_{\mathrm{XUV}}(\tau)=E_{\mathrm{XuV}}^{(0)}+\sum_{i=1}^{N} \Delta E_{i} \cos \left(\frac{2 \pi}{T_{i}} \tau+\varphi_{i}\right)
$$

where $E_{\mathrm{xuv}}^{(0)}$ is the photon energy position of the molecular ground state at equilibrium geometry, $\Delta E_{i}$ is the XUV photon energy excursion of the cosinusoidal oscillation, $T_{i}$ is the period of the oscillation, and $\varphi_{i}$ is the phase of the oscillation. The phases are reported as negative values in accordance with the convention adopted in Ref. ${ }^{16}$. The results of the sum of cosines fitting are plotted with red lines in Fig. 6, and the 


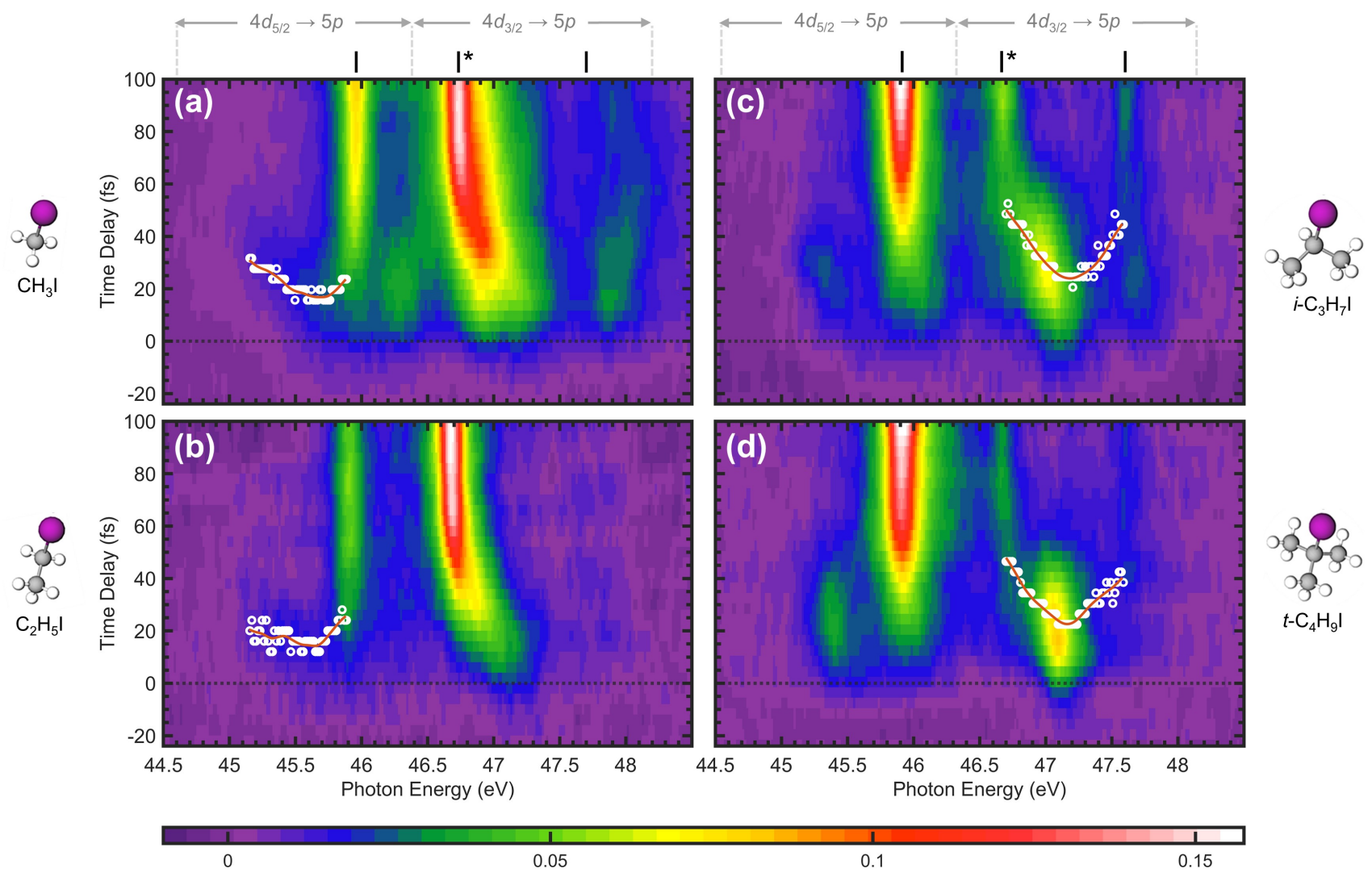

FIG. 5. Expanded view of conical intersection dynamics in the experimental (a) $\mathrm{CH}_{3} \mathrm{I}$, (b) $\mathrm{C}_{2} \mathrm{H}_{5} \mathrm{I}$, (c) $i$ - $\mathrm{C}_{3} \mathrm{H}_{7} \mathrm{I}$, and $(\mathrm{d}) t$ - $\mathrm{C}_{4} \mathrm{H}_{9} \mathrm{I}$ transients. Wave packet bifurcation at the ${ }^{3} \mathrm{Q}_{0} /{ }^{1} \mathrm{Q}_{1}$ conical intersection manifests as a u-shaped spectral feature in the $4 d_{5 / 2} \rightarrow 5 p$ spectral region of (a)-(b) and in the $4 d_{3 / 2} \rightarrow 5 p$ spectral region of (c)-(d). The conical intersection crossing time is designated as the minimum of a spline trace (orange curve) along the intensity maxima (white circles) of the feature.

\begin{tabular}{|cccccc|}
\hline Molecule & $\begin{array}{c}\text { Mode } \\
\text { assignment }\end{array}$ & Period $T(\mathrm{fs})$ & Frequency $\omega\left(\mathrm{cm}^{-1}\right)$ & Phase $\phi(\mathrm{rad})$ & $\begin{array}{c}\text { XUV photon energy } \\
\text { excursion } \Delta E(\mathrm{meV})\end{array}$ \\
\hline \hline $\mathrm{CH}_{3} \mathrm{I}$ & C-I stretch & $62 \pm 1$ & $538 \pm 7(533)^{\mathrm{a}}$ & $(-0.81 \pm 0.07) \pi$ & $130 \pm 16$ \\
\hline $\mathrm{C}_{2} \mathrm{H}_{5} \mathrm{I}$ & C-I stretch & $66 \pm 1$ & $505 \pm 7(512 \pm 10)^{\mathrm{b}}$ & $(-0.62 \pm 0.04) \pi$ & $140 \pm 10$ \\
& CCI bend & $119 \pm 4$ & $280 \pm 9(261 \pm 10)^{\mathrm{b}}$ & $(-0.87 \pm 0.12) \pi$ & $50 \pm 10$ \\
\hline$i-\mathrm{C}_{3} \mathrm{H}_{7} \mathrm{I}$ & C-I stretch & $67 \pm 1$ & $498 \pm 7(502 \pm 10)^{\mathrm{b}}$ & $(-0.69 \pm 0.05) \pi$ & $67 \pm 5$ \\
& CCI bend & $125 \pm 3$ & $267 \pm 6(262 \pm 10)^{\mathrm{b}}$ & $(-0.79 \pm 0.10) \pi$ & $32 \pm 5$ \\
\hline$t-\mathrm{C}_{4} \mathrm{H}_{9} \mathrm{I}$ & C-I stretch & $69 \pm 1$ & $483 \pm 7(496 \pm 10)^{\mathrm{b}}(-0.76 \pm 0.14) \pi$ & $32 \pm 7$ \\
& CCI bend & $129 \pm 2$ & $259 \pm 4(267 \pm 10)^{\mathrm{b}}$ & $(-0.89 \pm 0.07) \pi$ & $64 \pm 7$ \\
\hline
\end{tabular}

TABLE II. Sum of cosines analysis of the ground state vibrational dynamics captured in Fig. 6. Fundamental frequencies of the vibrational normal modes obtained from ${ }^{\mathrm{a}}$ Ref. $^{42}$ and ${ }^{\mathrm{b}}$ Ref. $^{43}$ are listed in parentheses.

parameters obtained from the fitting are summarized in Table II.

First, the measured phases are considered. Each cosinusoidal oscillation is assumed to be launched with a phase bounded by $-\pi<\varphi<-\pi / 2$. Oscillations initiated at the outer turning point and the equilibrium position of the ground state are associated with phases of $-\pi$ and $-\pi / 2$, respectively, as discussed previously in Ref. ${ }^{16}$. Ground state vibrational wave packets in all molecules are therefore considered to be launched between the outer turning point and equilibrium position, which is consistent with a RISRS launching mecha- nism.

Next, the measured periods of the oscillations are considered. The periodicities reveal the mode composition of the corresponding wave packets. For a superposition of $v=0$ and 1 levels of a particular vibrational mode, the wave packet oscillates with a periodicity given by $T=(c \omega)^{-1}$, where $c$ is the speed of light and $\omega$ corresponds to the fundamental vibrational frequency of the normal mode. ${ }^{12,15,16}$ In contrast, a superposition involving higher vibrational levels, for example $v=0$ and 2 or $v=1$ and 2 , will result in oscillatory frequencies that are either greater than or less than the fundamental 

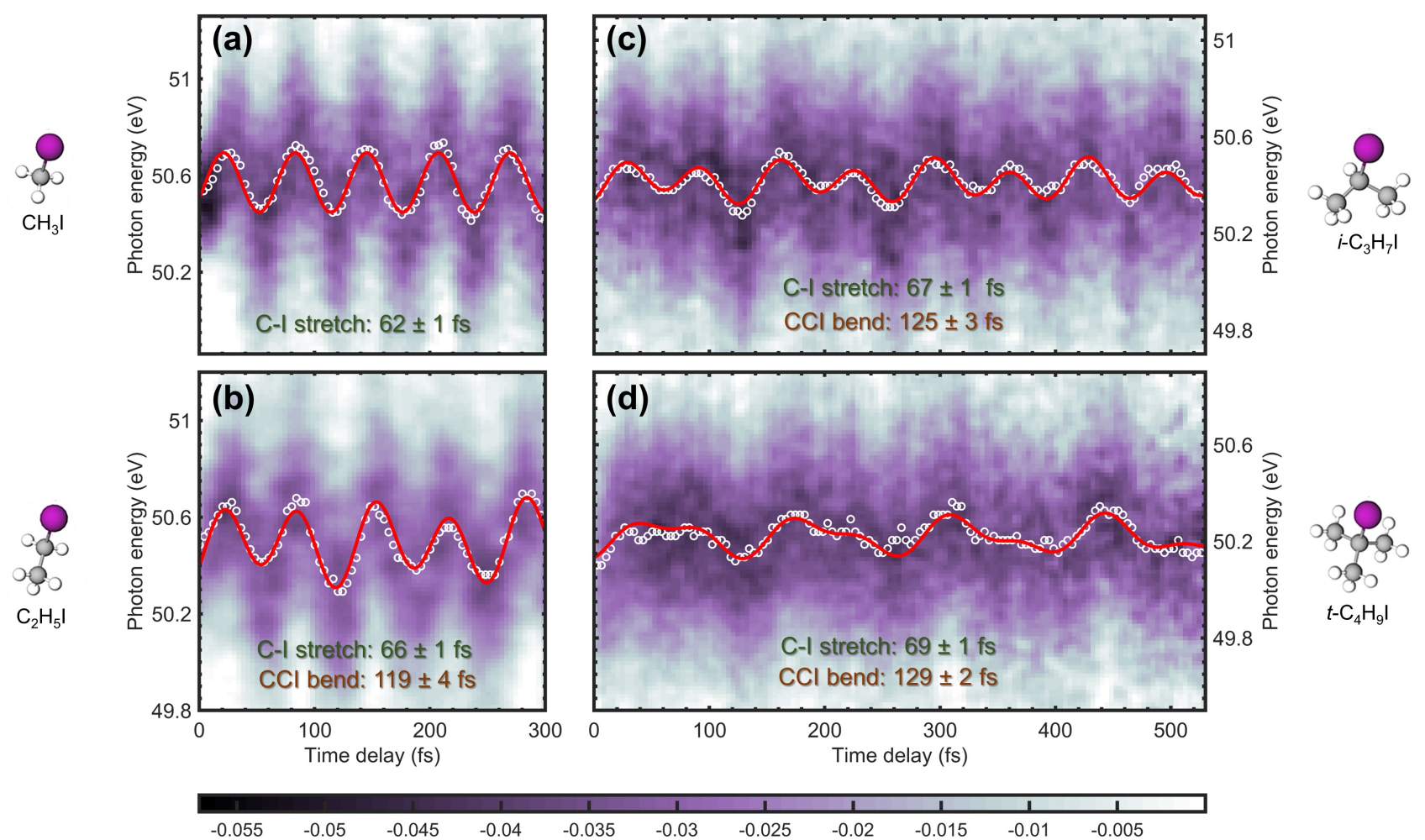

FIG. 6. Detection of ground state vibrational dynamics in (a) $\mathrm{CH}_{3} \mathrm{I}$, (b) $\mathrm{C}_{2} \mathrm{H}_{5} \mathrm{I}$, (c) $i-\mathrm{C}_{3} \mathrm{H}_{7} \mathrm{I}$, and (d) $t$ - $\mathrm{C}_{4} \mathrm{H}_{9} \mathrm{I}$. The transients plotted in (a)-(d) represent an expanded view of the oscillating ground state depletion signals in Fig. 2 associated with $4 d_{5 / 2} \rightarrow \sigma^{*}$ transitions. The photon energy position of the signal (white circles) is fit to a sum of cosines (red curve), allowing for identification of underlying vibrational modes.

frequency. ${ }^{16}$ In Table II, components of the ground state oscillations are assigned to fundamental vibrational frequencies of the molecules. As discussed in Ref. ${ }^{12}$, the ground state oscillation of $\mathrm{CH}_{3} \mathrm{I}$ is modeled by a single cosine whose periodicity is strongly consistent with a superposition of $v=0$ and 1 levels of the C-I stretch mode. In contrast, the ground state oscillations of $\mathrm{C}_{2} \mathrm{H}_{5} \mathrm{I}, i-\mathrm{C}_{3} \mathrm{H}_{7} \mathrm{I}$, and $t-\mathrm{C}_{4} \mathrm{H}_{9} \mathrm{I}$ are modeled by a sum of two cosines whose periodicities are consistent with the involvement of $v=0$ and 1 levels of the C-I stretch and CCI bend modes of the molecules. The detection of ground state motion along the C-I stretch mode in all molecules is consistent with rapid C-I separation along the repulsive $A$-band states. ${ }^{25}$ In addition, the detection of CCI bend modes in the ground state dynamics of $\mathrm{C}_{2} \mathrm{H}_{5} \mathrm{I}, i-\mathrm{C}_{3} \mathrm{H}_{7} \mathrm{I}$, and $t-\mathrm{C}_{4} \mathrm{H}_{9} \mathrm{I}$ molecules suggests that the R-group structure of the molecule rapidly planarizes on the excited state, causing a compression of the CCI bend angle. The structural dynamics of the substituted alkyl iodides near the conical intersection (Fig. 1a, Regions 1-2) are therefore characterized by multidimensional motion which includes not only C-I separation but also bending vibrations. The observations are consistent with previous resonance Raman spectroscopy experiments using narrowband UV lasers on the detection of $A$-band dynamics in alkyl iodides. ${ }^{18,43}$

The detected ground state vibrational modes may only re- flect a subset of the structural dynamics occurring in the excited state, due to potential limitations in preparing coherences among the ground state vibrational modes and in the XUV probing scheme. For example, high-frequency vibrations that may be involved in excited state dynamics might not appear in the ground state dynamics due to limitations in the duration of the driving RISRS pulse, ${ }^{40}$ as well as the time delay step size. The launching of a wave packet along a particular ground state normal mode through RISRS requires a driving pulse that is shorter in duration than the mode's oscillation period. Although the current UV pump pulse is shorter in duration compared to the majority of normal mode oscillation periods in the alkyl iodides, the pulse is insufficient for the preparation of a RISRS wave packet along high-frequency modes with sub-20 fs oscillation periods, such as $\mathrm{CH}_{3}$ rock and HCI bend vibrations. In addition, active ground state vibrations may lead to negligible XUV photon energy shifts, and thus the vibrations may not be experimentally detectable. However, while the modes detected may not provide a complete picture of all vibrational motions on the excited state, the detection of multiple vibrational modes in the substituted $\mathrm{C}_{2} \mathrm{H}_{5} \mathrm{I}, i-\mathrm{C}_{3} \mathrm{H}_{7} \mathrm{I}$, and $t-\mathrm{C}_{4} \mathrm{H}_{9} \mathrm{I}$ alkyl iodides provides evidence for the multidimensionality of vibrations in the $A$-band in the vicinity of the ${ }^{3} \mathrm{Q}_{0} /{ }^{1} \mathrm{Q}_{1}$ crossing.

Finally, we comment on the amplitude of the XUV photon 
energy excursions in the ground state oscillations. The excursions associated with the C-I stretch oscillation are observed to decrease with greater $\mathrm{CH}_{3}$ substitution of the molecules, whereas the excursions associated with the CCI bend oscillation are observed to increase. This trend may have several possible origins. One possibility is that differences in the gradients of the ground state potential or the core-excited $4 d_{j}-\sigma^{*}$ states between the alkyl iodides may lead to differences in the measured XUV transition energies. Another possibility for the increased excursion of CCI bend oscillations relative to $\mathrm{C}$-I stretch oscillations is that multidimensional motion along the excited states become increasingly important with greater substitution. The origin of the excursion trends could be clarified by future theoretical XUV spectrum or potential energy surface calculations.

\section{CONCLUSIONS}

Nonadiabiatic dynamics at conical intersections are characterized by the coupling of electronic and nuclear motion. In this work, ATAS combined with resonant $\sim 20$ fs UV pump pulses reveal electronic dynamics at a conical intersection as well as vibrational dynamics in the $A$-band photodissociation of alkyl iodides. Alkyl iodides of increasing structural complexity, $\mathrm{CH}_{3} \mathrm{I}, \mathrm{C}_{2} \mathrm{H}_{5} \mathrm{I}, i-\mathrm{C}_{3} \mathrm{H}_{7} \mathrm{I}$, and $t-\mathrm{C}_{4} \mathrm{H}_{9} \mathrm{I}$, are investigated. Owing to the short duration of the UV pump and the sensitivity of the XUV technique to molecular electronic and nuclear structures, $\sim 14-24$ fs wave packet bifurcation between distinct $A$-band electronic states at the ${ }^{3} \mathrm{Q}_{0} /{ }^{1} \mathrm{Q}_{1}$ and coherent vibrations launched by RISRS in the molecular ground state are observed. The ground state RISRS dynamics indirectly reflect vibrations on the excited states within the duration of the UV pump pulse, and are therefore expected to reflect vibrational activity near the conical intersection. For the substituted alkyl iodides studied, $\mathrm{C}_{2} \mathrm{H}_{5} \mathrm{I}, i-\mathrm{C}_{3} \mathrm{H}_{7} \mathrm{I}$, and $t-\mathrm{C}_{4} \mathrm{H}_{9} \mathrm{I}, \mathrm{C}$-I stretch as well as CCI bend motions are detected, providing evidence for excited state multidimensional vibrations. In conclusion, this work demonstrates the observation of both electronic and vibrational dynamics associated with a chemical reaction using ATAS. In the future, the combination of ATAS experiments with computational investigations of electronic-nuclear couplings, ${ }^{4,44,45}$ could provide mechanistic insights into the nonadiabatic dynamics governing chemical processes and an avenue for examining the effects of chemical substitution on such dynamics.

\section{SUPPLEMENTARY MATERIAL}

See the supplementary material for further details on the experimental methods, raw and post-processed transient spectra plotted up to $60 \mathrm{eV}$ photon energy, and calculations of the I:I* ratios.

\section{ACKNOWLEDGMENTS}

This work has been financed by the National Science Foundation (NSF) (Grant No. CHE-1951317 and No. CHE-1660417) (K.F.C., S.R.L.) and by the U.S. Army Research Office (ARO) under Grant No. W911NF-14-1-0383 (K.F.C., D.M.N., S.R.L.) and under Grant No. W911NF20-1-0127 (K.F.C., D.M.N.). J.G.V. was financed by Project PID2019-106732GB-I00 of the Ministerio de Ciencia, Innovación y Universidades of Spain. L.B. was financed in part by the Spanish State Research Agency (AEI/10.13039/501100011033), Grant PGC2018-096444-BI00, and the Madrid Regional Government through the program Proyectos Sinérgicos de I + D (Grant Y2018/NMT-5028 FULMATEN-CM). Computational work by H.W. and D.P. was performed within the Gas Phase Chemical Physics Program through the Chemical Sciences Division of Lawrence Berkeley National Laboratory (LBNL), supported by the U.S. Department of Energy (DOE), Office of Science, Office of Basic Energy Sciences, under Contract No. DEAC02-05CH11231. Supercomputer time was provided by the National Energy Research Scientific Computing Center. S.M.P acknowledges funding from the European Unions Horizon 2020 Research and Innovation Program under the Marie Sklodowska-Curie grant agreement (No. 842539, ATTOCONTROL). Additionally, we thank Dr. Maurizio Reduzzi for his contributions to the construction of the UV pump pulse setup.

\section{DATA AVAILABILITY STATEMENT}

The data that support the findings of this study are available from the corresponding author upon reasonable request.

${ }^{1}$ D. R. Yarkony, "Conical intersections: the new conventional wisdom," J. Phys. Chem. A 105, 6277-6293 (2001).

${ }^{2}$ W. Domcke and D. R. Yarkony, "Role of conical intersections in molecular spectroscopy and photoinduced chemical dynamics," Annu. Rev. Phys. Chem. 63, 325-352 (2012).

${ }^{3}$ F. Lépine, M. Y. Ivanov, and M. J. J. Vrakking, "Attosecond molecular dynamics: fact or fiction?" Nat. Photonics 8, 195-204 (2014).

${ }^{4}$ M. S. Schuurman and A. Stolow, "Dynamics at conical intersections," Annu. Rev. Phys. Chem. 69, 427-450 (2018).

${ }^{5}$ H. Ibrahim, B. Wales, S. Beaulieu, B. E. Schmidt, N. Thiré, E. P. Fowe, É. Bisson, C. T. Hebeisen, V. Wanie, M. Giguére, J.-C. Kieffer, M. Spanner, A. D. Bandrauk, J. Sanderson, M. S. Schuurman, and F. Légaré, "Tabletop imaging of structural evolutions in chemical reactions demonstrated for the acetylene cation," Nature Communications 5, 4422 (2014).

${ }^{6}$ K. S. Zinchenko, F. Ardana-Lamas, I. Seidu, S. P. Neville, J. van der Veen, V. U. Lanfaloni, M. S. Schuurman, and H. J. Wörner, "Sub-7-femtosecond conical-intersection dynamics probed at the carbon k-edge," Science 371, 489-494 (2021).

${ }^{7}$ L. Bäuml, T. Schnappinger, M. F. Kling, and R. de Vivie-Riedle, "Photoinduced coupled nuclear and electron dynamics in the nucleobase uracil," Frontiers in Physics 9 (2021).

${ }^{8}$ L. Young, K. Ueda, M. Gühr, P. H. Bucksbaum, M. Simon, S. Mukamel, N. Rohringer, K. C. Prince, C. Masciovecchio, M. Meyer, A. Rudenko, D. Rolles, C. Bostedt, M. Fuchs, D. A. Reis, R. Santra, H. Kapteyn, M. Murnane, H. Ibrahim, F. Légaré, M. Vrakking, M. Isinger, D. Kroon, M. Gisselbrecht, A. L'Huillier, H. J. Wörner, and S. R. Leone, "Roadmap of ultrafast X-ray atomic and molecular physics," J. Phys. B 51, 032003 (2018). 
${ }^{9}$ H. Timmers, X. Zhu, Z. Li, Y. Kobayashi, M. Sabbar, M. Hollstein, M. Reduzzi, T. J. Martínez, D. M. Neumark, and S. R. Leone, "Disentangling conical intersection and coherent molecular dynamics in methyl bromide with attosecond transient absorption spectroscopy," Nat. Commun. 10, 3133 (2019).

${ }^{10}$ Y. Kobayashi, K. F. Chang, T. Zeng, D. M. Neumark, and S. R. Leone, "Direct mapping of curve-crossing dynamics in IBr by attosecond transient absorption spectroscopy," Science 365, 79-83 (2019).

${ }^{11}$ K. F. Chang, M. Reduzzi, H. Wang, S. M. Poullain, Y. Kobayashi, L. Barreau, D. Prendergast, D. M. Neumark, and S. R. Leone, "Revealing electronic state-switching at conical intersections in alkyl iodides by ultrafast XUV transient absorption spectroscopy," Nat. Commun. 11, 4042 (2020).

${ }^{12}$ K. F. Chang, H. Wang, S. M. Poullain, D. Prendergast, D. M. Neumark, and S. R. Leone, "Mapping wave packet bifurcation at a conical intersection in $\mathrm{CH}_{3} \mathrm{I}$ by attosecond XUV transient absorption spectroscopy," J. Chem. Phys. 154, 234301 (2021).

${ }^{13}$ F. Rott, M. Reduzzi, T. Schnappinger, Y. Kobayashi, K. F. Chang, H. Timmers, D. M. Neumark, R. d. Vivie-Riedle, and S. R. Leone, "Ultrafast strong-field dissociation of vinyl bromide: An attosecond transient absorption spectroscopy and non-adiabatic molecular dynamics study," Structural Dynamics 8, 034104 (2021).

${ }^{14}$ V. Scutelnic, S. Tsuru, M. Pápai, Z. Yang, M. Epshtein, T. Xue, E. Haugen, Y. Kobayashi, A. I. Krylov, K. B. Møller, S. Coriani, and S. R. Leone, "Xray transient absorption reveals the $1 \mathrm{Au}\left(\mathrm{n} \pi^{*}\right)$ state of pyrazine in electronic relaxation," Nature Communications 12, 5003 (2021).

${ }^{15}$ E. R. Hosler and S. R. Leone, "Characterization of vibrational wave packets by core-level high-harmonic transient absorption spectroscopy," Phys. Rev. A 88, 023420 (2013).

${ }^{16}$ Z. Wei, J. Li, L. Wang, S. T. See, M. H. Jhon, Y. Zhang, F. Shi, M. Yang, and Z.-H. Loh, "Elucidating the origins of multimode vibrational coherences of polyatomic molecules induced by intense laser fields," Nat. Commun. 8, 735 (2017).

${ }^{17}$ Z. Wei, L. Tian, J. Li, Y. Lu, M. Yang, and Z.-H. Loh, "Tracking ultrafast bond dissociation dynamics at $0.1 \AA$ resolution by femtosecond extreme ultraviolet absorption spectroscopy,' J. Phys. Chem. Lett. 9, 5742-5747 (2018).

${ }^{18}$ D. L. Phillips, A. B. Myers, and J. J. Valentini, "Investigation of solvation effects on short-time photodissociation dynamics of alkyl iodides," J. Phys. Chem. 96, 2039-2044 (1992).

${ }^{19}$ C. M. Roehl, J. B. Burkholder, G. K. Moortgat, A. R. Ravishankara, and P. J. Crutze, "Temperature dependence of UV absorption cross sections and atmospheric implications of several alkyl iodides,' J. Geophys. Res. 102, 12819-12829 (1997).

${ }^{20}$ R. S. Mulliken, "Intensities in molecular electronic spectra X. calculations on mixed-halogen, hydrogen halide, alkyl halide, and hydroxyl spectra," J. Chem. Phys. 8, 382-395 (1940).

${ }^{21}$ S. H. Gardiner, M. L. Lipciuc, T. N. V. Karsili, M. N. R. Ashfold, and C. Vallance, "Dynamics of the A-band ultraviolet photodissociation of methyl iodide and ethyl iodide via velocity-map imaging with 'universal' detection,” Phys. Chem. Chem. Phys. 17, 4096-4106 (2015).

${ }^{22}$ A. Gedanken and M. D. Rowe, "Magnetic circular dichroism spectra of the methyl halides: resolution of the $\mathrm{n} \rightarrow \sigma^{*}$ continuum," Chem. Phys. Lett. 34, 39-43 (1975).

${ }^{23}$ A. Gedanken, "The magnetic circular dichroism of the $\mathrm{A}$ band in $\mathrm{CF}_{3} \mathrm{I}$, $\mathrm{C}_{2} \mathrm{H}_{5} \mathrm{I}$, and t-BuI," Chem. Phys. Lett. 137, 462-466 (1987).

${ }^{24}$ F. Godwin, P. A. Gorry, P. M. Hughes, D. Raybone, T. M. Watkinson, and J. Whitehead, "Two-photon VUV laser-induced fluorescence detection of $\mathrm{I}^{*}\left({ }^{2} \mathrm{P}_{1 / 2}\right)$ and $\mathrm{I}\left({ }^{2} \mathrm{P}_{3 / 2}\right)$ from alkyl iodide photodissociation at $248 \mathrm{~nm}$," Chem. Phys. Lett. 135, 163-169 (1987).

${ }^{25}$ M. E. Corrales, V. Loriot, G. Balerdi, J. González-Vázquez, R. de Nalda, L. Bañares, and A. H. Zewail, "Structural dynamics effects on the ultrafast chemical bond cleavage of a photodissociation reaction," Phys. Chem. Chem. Phys. 16, 8812-8818 (2014).
${ }^{26}$ E. M. Warne, B. Downes-Ward, J. Woodhouse, M. A. Parkes, E. Springate, P. A. J. Pearcy, Y. Zhang, G. Karras, A. S. Wyatt, R. T. Chapman, and R. S. Minns, "Photodissociation dynamics of methyl iodide probed using femtosecond extreme ultraviolet photoelectron spectroscopy," Phys. Chem. Chem. Phys. 22, 25695-25703 (2020).

${ }^{27}$ Y. S. Kim, W. K. Kang, D.-C. Kim, and K.-H. Jung, "Photodissociation of tert-butyl iodide at 277 and $304 \mathrm{~nm}$ : Evidence for direct and indirect dissociation in A-band photolysis of alkyl iodide," J. Phys. Chem. A 101, 7576-7581 (1997).

${ }^{28}$ D. Zhong and A. H. Zewail, "Femtosecond real-time probing of reactions. 23 studies of temporal, velocity, angular, and state dynamics from transition states to final products by femtosecond-resolved mass spectrometry," J. Phys. Chem. A 102, 4031-4058 (1998).

${ }^{29}$ K. Kavita and P. K. Das, "Dynamics of $\mathrm{I}^{*}\left({ }^{2} \mathrm{P}_{1 / 2}\right)$ production from fluorinated alkyl iodides at 266, 280, and $2305 \mathrm{~nm}$," J. Chem. Phys. 112, 84268431 (2000).

${ }^{30}$ Y. Tang, W.-B. Lee, Z. Hu, B. Zhang, and K.-C. Lin, "Productions of I, I*, and $\mathrm{C}_{2} \mathrm{H}_{5}$ in the A-band photodissociation of ethyl iodide in the wavelength range from 245 to $283 \mathrm{~nm}$ by using ion-imaging detection," J. Chem. Phys. 126, 064302 (2007).

${ }^{31}$ H. Timmers, Y. Kobayashi, K. F. Chang, M. Reduzzi, D. M. Neumark, and S. R. Leone, "Generating high-contrast, near single-cycle waveforms with third-order dispersion compensation," Opt. Lett. 42, 811-814 (2017).

${ }^{32}$ A. R. Attar, A. Bhattacherjee, and S. R. Leone, "Direct observation of the transition-state region in the photodissociation of $\mathrm{CH}_{3} \mathrm{I}$ by femtosecond extreme ultraviolet transient absorption spectroscopy,' J. Phys. Chem. Lett. 6, 5072-5077 (2015).

${ }^{33}$ H. Wang, M. Odelius, and D. Prendergast, "A combined multi-reference pump-probe simulation method with application to XUV signatures of ultrafast methyl iodide photodissociation," J. Chem. Phys. 151, 124106 (2019).

${ }^{34}$ M. Pettini, M. Mazzoni, and G. P. Tozzi, "Excitation of the inner 4d shell of neutral iodine," Phys. Lett. A 82, 168-170 (1981).

${ }^{35}$ G. O’Sullivan, C. McGuinness, J. T. Costello, E. T. Kennedy, and B. Weinmann, "Trends in 4d-subshell photoabsorption along the iodine isonuclear sequence: I, I ${ }^{+}$, and $\mathrm{I}^{2+}$," Phys. Rev. A 53, 3211-3226 (1996).

${ }^{36} \mathrm{~S}$. Uma and P. K. Das, "Production of $\mathrm{I}^{*}\left({ }^{2} \mathrm{P}_{1 / 2}\right)$ in the ultraviolet photodissociation of $\alpha$-branched alkyl iodides," J. Chem. Phys. 104, 4470-4474 (1996).

${ }^{37}$ Y. Yan, E. B. Gamble, and K. A. Nelson, "Impulsive stimulated scattering: General importance in femtosecond laser pulse interactions with matter, and spectroscopic applications,” J. Chem. Phys. 83, 5391-5399 (1985).

${ }^{38}$ A. M. Walsh and R. F. Loring, "Theory of resonant and nonresonant impulsive stimulated raman scattering," Chem. Phys. Lett. 160, 299-304 (1989).

${ }^{39}$ U. Banin, A. Bartana, S. Ruhman, and R. Kosloff, "Impulsive excitation of coherent vibrational motion ground surface dynamics induced by intense short pulses," J. Chem. Phys. 101, 8461-8481 (1994).

${ }^{40}$ L. Dhar, J. A. Rogers, and K. A. Nelson, "Time-resolved vibrational spectroscopy in the impulsive limit," Chem. Rev. 94, 157-193 (1994).

${ }^{41}$ M. T. Zanni, B. J. Greenblatt, A. V. Davis, and D. M. Neumark, "Photodissociation of gas phase $\mathrm{I}_{3}$ using femtosecond photoelectron spectroscopy," J. Chem. Phys. 111, 2991-3003 (1999).

${ }^{42}$ T. Shimanouchi, Tables of Molecular Vibrational Frequencies, Vol. I (National Bureau of Standards, 1972).

${ }^{43}$ D. L. Phillips, B. A. Lawrence, and J. J. Valentini, "Substituent effects on gas-phase photodissociation dynamics: Resonance Raman spectra of ethyl iodide, isopropyl iodide, and tert-butyl iodide," J. Phys. Chem. 95, 90859091 (1991).

${ }^{44}$ S. P. Neville, Y. Wang, A. E. Boguslavskiy, A. Stolow, and M. S. Schuurman, "Substituent effects on dynamics at conical intersections: allene and methyl allenes," J. Chem. Phys. 144, 014305 (2016).

${ }^{45}$ R. J. MacDonell, M. E. Corrales, A. E. Boguslavskiy, L. Bañares, A. Stolow, and M. S. Schuurman, "Substituent effects on nonadiabatic excited state dynamics: Inertial, steric, and electronic effects in methylated butadienes," J. Chem. Phys. 152, 084308 (2020). 